\title{
Pandemia da COVID-19 na região Entre Douro e Minho: qual o impacto do confinamento na qualidade do ar?
}

\author{
https://doi.org/10.21814/uminho.ed.24.16
}

\section{Paula Marinho Reis}

Paula Marinho Reis (ORCID: 0000-0003-0922-8290) é Professora Auxiliar no Departamento de Ciências da Terra da Escola de Ciências da Universidade do Minho, tendo-se formado em Engenharia Geológica na Universidade de Aveiro, onde obteve também o seu doutoramento em Geociências. A sua investigação insere-se no domínio da geoquímica ambiental e saúde humana. É editora coordenadora da revista Environmental Geochemistry and Health, publicada pela Elsevier e integra o Regional Board for Europe da Society for Environmental Geochemistry and Health. 


\section{RESUMO}

A 11 de março de 2020, a Organização Mundial da Saúde declarou que a doença COVID-19 havia sido caracterizada como uma pandemia. Devido à rápida propagação do vírus, a 18 de março, o Presidente da República decreta o estado de emergência por 15 dias, o qual viria a ser prolongado até ao dia 2 de maio. Com o dever de recolhimento domiciliário, quase todas as atividades industriais e transportes em massa foram proibidos. Após cerca de seis semanas de confinamento obrigatório, o desconfinamento viria a ser implementado em três fases.

A aglomeração Entre Douro e Minho contém seis estaçôes de medição da qualidade do ar distribuídas pelas cidades de Braga, Guimarães, Santo Tirso, Paços de Ferreira e Paredes. Neste trabalho foram analisados com maior detalhe os poluentes medidos cuja monitorização é obrigatória, isto é, dióxido de azoto, matéria particulada de diâmetro inferior a $10 \mu \mathrm{m}$ e ozono, com o monóxido de azoto e os óxidos de azoto a serem utilizados essencialmente como auxiliares na interpretação dos resultados. A severa limitação do movimento de pessoas após o confinamento e subsequente desconfinamento resultaram numa redução significativa da concentração de dióxido de azoto atmosférico, principalmente devido ao tráfego de veículos. Apesar da redução significativa deste poluente nas fases confinamento e desconfinamento, o ozono apresentou um aumento significativo, provavelmente devido à menor concentração de monóxido de azoto atmosférico. No entanto, são necessários dados adicionais para realizar uma avaliação mais precisa.

\section{INTRODUÇÃO}

A poluição atmosférica é responsável por um elevado número de mortes assim como um aumento da incidência de doenças respiratórias (Dutheil et al., 2020). De acordo com a Organização Mundial da Saúde (OMS), 4,6 milhóes de pessoas morrem anualmente de doenças diretamente relacionadas com a má qualidade do ar (Cohen et al., 2017). Enfermidades associadas à poluição atmosférica incluem, entre outras, agravamento da asma, bronquite, enfisema pulmonar, doenças cardíacas e alergias respiratórias (Brauer, 2010). 
Matéria particulada (PM - particulate matter) incluindo $\mathrm{PM}_{10}$ (partículas com diâmetro aerodinâmico $£ 10 \mu \mathrm{m})$, dióxido de azoto $\left(\mathrm{NO}_{2}\right)$ e dióxido de enxofre $\left(\mathrm{SO}_{2}\right)$ são poluentes atmosféricos muito comuns em ambientes urbanos e vários estudos realizados demonstraram já o seu impacto na saúde humana (Otomani et al., 2020). Estes poluentes são emitidos por fontes antrópicas, incluindo tráfego rodoviário, queima de biomassa e atividades industriais (Song et al., 2017; He et al., 2020). Além destas fontes de emissão, a poluição de longo alcance também desempenha um papel importante nalguns países do mundo.

A atual legislação europeia relativa à qualidade do ar baseia-se no princípio de que os Estados-Membros da União Europeia (EU) dividem os seus territórios num conjunto de zonas de gestão onde os países são obrigados a avaliar a qualidade do ar através de sistemas de medição ou modelação (AEA, 2016). Em Portugal, o Decreto-Lei no 102/2010, de 23 de setembro de 2010, posteriormente alterado pelo Decreto-Lei no 43/2015, de 27 de março, estabelece os valores limite (VL) para as emissóes poluentes no território (APA, 2020). No continente, existe em funcionamento um total de 64 estações de medição da qualidade do ar, seis das quais localizadas na denominada aglomeração Entre Douro e Minho. A base de dados QualAr, promovida pela Agência Portuguesa do Ambiente (APA), centraliza todos os dados de qualidade do ar medidos em Portugal.

A COVID-19 é uma doença altamente contagiosa identificada pela primeira vez em Wuhan, na China Central, em dezembro de 2019. A 11 de março de 2020, a OMS declarou que a doença COVID-19, causada pelo novo Coronavírus SARS-CoV-2, havia sido caracterizada como uma pandemia (OMS, 2020). A 2 de março, a Ministra da Saúde havia anunciado os dois primeiros casos de pessoas infetadas com o novo coronavírus no nosso país e a 7 de março o Reitor da Universidade do Minho encerra o Instituto de Ciências Sociais, situado no campus universitário de Gualtar. Devido à rápida propagação do vírus, a 18 de março, o Presidente da República decreta o estado de emergência por 15 dias, o qual viria a ser prolongado até ao dia 2 de maio. Com o dever de recolhimento domiciliário, quase todas as atividades industriais e transportes em massa foram proibidos. Após cerca de seis semanas de confinamento obrigatório, o desconfinamento viria a ser implementado em três fases, a primeira com início a 
4 de maio, a segunda a 18 de maio e a última a 1 de junho, com um retomar gradual das atividades anteriormente suspensas.

Estudos disponíveis na literatura têm vindo a reportar uma diminuição sem precedentes no nível de poluição atmosférica, durante as duras fases de confinamento impostas em cidades como Milão, Itália (Collivignarelli et al., 2020), Deli, Índia (Mahato et al., 2020) ou S. Paulo, Brasil (Nakada \& Urban, 2020).

Este artigo tem como objetivo estudar o impacto do confinamento imposto pela pandemia da COVID-19 na qualidade do ar na região Entre Douro e Minho.

\section{A ÁREA DE ESTUDO}

A aglomeração Entre Douro e Minho contém seis estações de medição da qualidade do ar, duas das quais localizadas na cidade de Braga, uma em contexto urbano (Avenida Frei Bartolomeu Mártires - S. Vítor) e a outra em ambiente rural (Frossos). As restantes estaçôes situam-se nas cidades de Guimarães, Santo Tirso, Paços de Ferreira, e Paredes.

Demograficamente são cidades com características diferentes, nomeadamente em termos de população residente, taxa de crescimento anual e densidade populacional (Tabela 1, Fonte: INE, 2016). Braga e Guimarães são as duas maiores cidades, com uma população residente de 182176 e 158048 habitantes, respetivamente. Embora menor em termos de tamanho da população, Paços de Ferreira destaca-se pela elevada densidade populacional (799.60 habitantes $/ \mathrm{km}^{2}$ ), superada apenas pela cidade de Braga com uma densidade populacional de quase 1000 habitantes $/ \mathrm{km}^{2}$. Em 2011, apenas Braga, Paços de Ferreira e Paredes apresentavam uma taxa de crescimento anual efetiva positiva (Tabela 1 ). 
Tabela 1. Dados territoriais das cidades incluídas na aglomeração Entre Douro e Minho.

$\begin{array}{lccc}\text { NUTS 2002 }^{\mathrm{a}} & \text { Densidade populacional }^{\mathrm{a}} & \text { População residente }^{\mathrm{a}} & \text { Taxa de } \text { crescimento }^{\mathrm{a}} \\ & \text { No/ km } & & \% \\ \text { Braga } & 993.30 & \text { No } & 0.38 \\ \text { Guimarães } & 655.70 & 182176 & -0.10 \\ \text { Santo Tirso } & 523.10 & 7148048 & -0.23 \\ \text { Paços de Ferreira } & 799.60 & 56769 & 0.74 \\ \text { Paredes } & 556.00 & 87159 & 0.36\end{array}$

${ }^{2}$ A classificação territorial utilizada corresponde à geografia em vigor à data dos Censos 2011; ${ }^{b}$ Os dados da população correspondem aos Censos 2011.

De acordo com a Nomenclatura de Unidades Territoriais para Fins Estatísticos (NUTS), a área de estudo localiza-se na Região Norte (NUTS III) de Portugal Continental (NUTS I), pertencendo Braga à sub-região do Cávado, Guimarães à sub-região do Ave, Paredes e Santo Tirso à sub-região da Área Metropolitana do Porto, e Paços de Ferreira à sub-região de Tâmega e Sousa (INE 2016).

O clima da região apresenta características atlânticas de transição para mediterrânicas e continentais, com veróes frescos e pouco secos no litoral e mais quentes a secos no interior (temperaturas médias mensais entre os $18^{\circ} \mathrm{C}$ e os $22^{\circ} \mathrm{C}$ ) e invernos suaves (temperaturas médias mensais entre os $8^{\circ} \mathrm{C}$ e os $12^{\circ} \mathrm{C}$ ). Segundo a classificação de Köppen-Geiger, a área insere-se numa zona de clima temperado com inverno chuvoso e verão seco e temperado (Csb) (IPMA 2020).

Atualmente, as seis estações de monitorização estão em operação, com capacidade para monitorizar e registar as concentrações de alguns poluentes. Os locais de implantação das estaçôes de medição estão indicados na figura 1. 


\section{MATERIAIS E MÉTODOS}

Para avaliar o estado da qualidade do ar durante o período de confinamento, foram analisados dados das seis estaçóes de monitoramento da qualidade do ar da aglomeração de Entre Douro e Minho (Figura 1).

Médias diárias de concentração de poluentes atmosféricos, incluindo $\mathrm{PM}_{10}$, óxidos de azoto $\left(\mathrm{NO}_{\mathrm{x}}\right)$, monóxido de azoto $(\mathrm{NO}), \mathrm{NO}_{2}$, e ozono $\left(\mathrm{O}_{3}\right)$, para os primeiros seis meses dos anos de 2019 e 2020, foram fornecidas pela Comissão de Coordenação e Desenvolvimento Regional do Norte (CCDR-N) As médias diárias foram calculadas a partir das médias horárias, com exceção do $\mathrm{O}_{3}$, cujas médias diárias correspondem a médias de $8 \mathrm{~h}$.

Note-se que, atualmente, as diferentes estações medem poluentes distintos, pelo que nem sempre foi possível efetuar uma análise global das seis estações de monitorização.

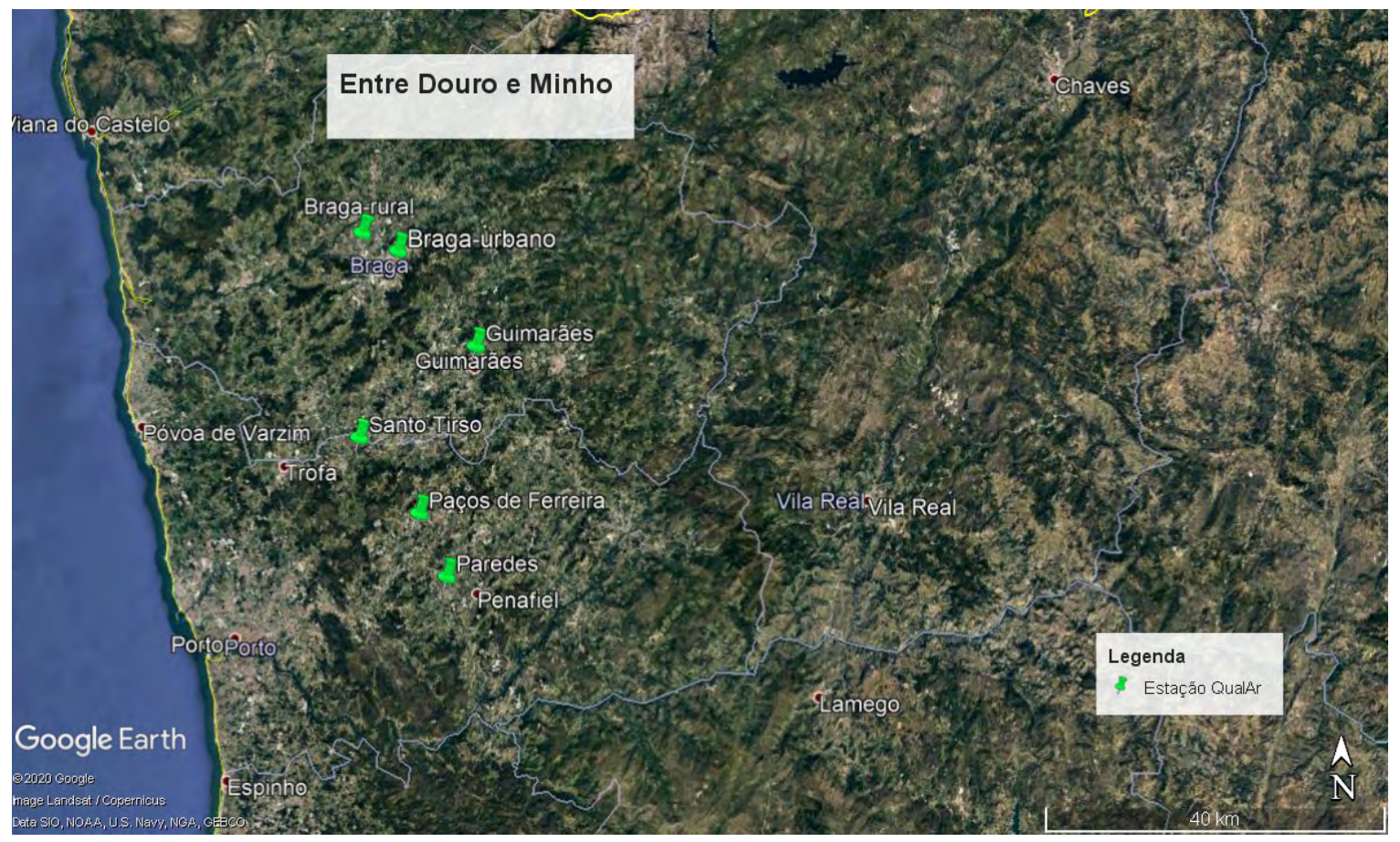

Figura 1. Localização das seis estações de medição da qualidade do ar, assinaladas na imagem com os símbolos verdes (imagem Google Earth, @ 2020 Google LLC). 
Os poluentes gasosos contribuem significativamente para as variações na química da atmosfera e resultam essencialmente da combustão de combustíveis fósseis. Os $\mathrm{NO}_{\mathrm{x}}$ são emitidos como $\mathrm{NO}$ o qual, na atmosfera, reage rapidamente com $\mathrm{O}_{3}$ ou radicais livres, formando $\mathrm{NO}_{2}$. As principais fontes antrópicas são fontes de combustão, móveis ou estacionárias. Além disso, nas camadas inferiores da atmosfera, o $\mathrm{O}_{3}$ é formado por uma série de reaçôes envolvendo $\mathrm{NO}_{\mathrm{x}}$ e compostos orgânicos voláteis, um processo que é iniciado pela luz solar (Awasthi 2016; Sicard et al., 2020). PM é o termo genérico usado para um tipo de poluentes atmosféricos, que consiste em misturas complexas e variáveis de partículas suspensas no ar respirável, que variam em tamanho e composição, e são produzidas por uma variedade de atividades naturais e antrópicas. As principais fontes de poluição por PM são fábricas, incineradoras de resíduos, tráfego rodoviário, atividades de construção civil, queima de biomassa e poeira natural ressuspensa por ação do vento (normalmente designadas por poeiras fugitivas). Apenas partículas com diâmetro aerodinâmico inferior a $10 \mu \mathrm{m}$ têm potencial para se depositarem na região traqueo-brônquica e alveolar e, portanto, apresentam o maior risco para a saúde humana quando inaladas (Kastury et al., 2017).

A Tabela 2 sumariza os poluentes monitorizados nas diferentes estações, assim como valores de eficiência calculados para os períodos em análise, de acordo com:

$$
\text { Eficiência }=\frac{N^{\circ} \text { de dados registado pelo sensor }}{N^{0} \text { total de dados }} \times 100 \%
$$

Neste trabalho são analisados com maior detalhe os poluentes cuja monitorização é obrigatória, isto é, $\mathrm{NO}_{2}, \mathrm{PM}_{10}$ e $\mathrm{O}_{3}$, com $\mathrm{NO}$ e $\mathrm{NO}_{\mathrm{x}}$ a serem utilizados essencialmente como auxiliares na interpretação dos resultados. Nenhuma das seis estações efetua medições de $\mathrm{SO}_{2}$ e monóxido de carbono $(\mathrm{CO})$, apesar da sua monitorização estar indicada na legislação.

As estações de Braga-urbano, Guimarães e Paredes não efetuam medições de $\mathrm{O}_{3}$, enquanto que a estação de Braga-rural não monitoriza o $\mathrm{NO}_{2}$. Entre janeiro de $2019 \mathrm{e}$ junho de 2020, a estação de Braga-urbano não efetuou mediçóes de $\mathrm{PM}_{10}$, e entre janeiro e junho de 2020 a estação de Paredes não monitorizou o $\mathrm{NO}_{2}$, provavelmente por avaria dos sensores. 
É de notar que, dos 182 dias que correspondem ao período janeiro-junho (no total de dados), em Braga-urbano apenas existem dados de $\mathrm{NO}_{2}$ para 119 dias (Eficiência $=65 \%$ ) em 2019 e 84 dias (Eficiência $=46 \%$ ) em 2020. Destacam-se eficiências baixas também para $\mathrm{PM}_{10}$ em Santo Tirso e Paços de Ferreira.

Para identificar os diferentes períodos utilizados na análise dos padróes de variação temporal dos poluentes, foram tomadas em consideração as medidas legislativas emitidas pelas autoridades. O período compreendido entre 1 de janeiro e 18 de março foi classificado como a fase anterior ao confinamento (AC). O período de 19 de março a 3 maio, no qual vigorou o Estado de Emergência, foi categorizado como o período de confinamento total (CT). As 3 fases de desconfinamento, entre 4 de maio e 1 de junho, foram identificadas como indicativas de um confinamento parcial (CP) devido ao levantamento de medidas mais rígidas que haviam levado ao encerramento de quase todas as atividades económicas e à restriçãao da circulação de pessoas. Finalmente, o período compreendido entre 2 e 30 de junho foi classificado como a fase de pós-confinamento (PC).

Tabela 2. Valores de eficiência calculados para os períodos janeiro-junho de 2019 e de 2020 nas diferentes estações de medição da qualidade do ar.

$\begin{array}{lllllll}\text { Estaçóes } & \text { Ano } & \mathrm{NO} & \mathrm{NO}_{2} & \mathrm{NO}_{\mathbf{x}} & \mathrm{O}_{3} & \mathbf{P M}_{10} \\ \text { Santo Tirso } & 2019 & 82 & 82 & 82 & 98 & 36 \\ & 2020 & 93 & 93 & 93 & 100 & 17 \\ \text { Braga (urbano) } & 2019 & 65 & 65 & 65 & \mathrm{a} & \mathrm{b} \\ & 2020 & 46 & 46 & 46 & \mathrm{a} & \mathrm{b} \\ \text { Braga (rural) } & 2019 & \mathrm{a} & \mathrm{a} & \mathrm{a} & 71 & 66 \\ & 2020 & \mathrm{a} & \mathrm{a} & \mathrm{a} & 100 & \mathrm{~b} \\ \text { Guimarães } & 2019 & 47 & 47 & 47 & \mathrm{a} & 72 \\ & 2020 & 88 & 88 & 88 & \mathrm{a} & 96 \\ \text { Paços de Ferreira } & 2019 & 84 & 84 & 84 & 41 & 62 \\ & 2020 & 46 & 46 & 46 & 100 & 63 \\ \text { Paredes } & 2019 & 69 & 69 & 69 & \mathrm{a} & 97 \\ & 2020 & \mathrm{~b} & \mathrm{~b} & \mathrm{~b} & \mathrm{a} & 75\end{array}$


Diferenças entre os grupos foram testadas usando o teste Mann - Whitney U, sendo os resultados interpretados com base nas diferenças de classificação ou nas medianas dos grupos. Uma probabilidade de 0,05 $(p<0.05)$ ou menor foi considerada significativa ao testar a hipótese nula de que não existem diferenças nas concentrações dos poluentes nos dois períodos considerados (Marinho-Reis et al., 2018).

\section{RESULTADOS E DISCUSSÃO}

\section{Distribuição das concentraçóes dos poluentes}

Potenciais diferenças na distribuição das concentrações dos poluentes foram avaliadas por comparação com as concentrações medidas nos primeiros seis meses de 2019.

$\mathrm{NO}_{2}$

$\mathrm{O} \mathrm{NO}_{2}$ é um gás com propriedades oxidantes capazes de contaminar o ar ambiente em muitos contextos urbanos e industriais. O potencial impacto da exposição humana a este poluente atmosférico inclui diversas doenças do trato respiratório, como por exemplo a asma (Nitschke 1999).

Tabela 3. Estatísticos sumários calculados para os dados de $\mathrm{NO}_{2}$ referentes aos primeiros seis meses de 2019 e 2020.

\begin{tabular}{|c|c|c|c|c|c|c|c|c|}
\hline \multirow[t]{2}{*}{$\mathrm{NO}_{2} \mu \mathrm{g} / \mathrm{m}^{3}$} & \multicolumn{2}{|c|}{ Mínimo } & \multicolumn{2}{|c|}{ Média \pm Desvio Padrão } & \multicolumn{2}{|c|}{ Mediana } & \multicolumn{2}{|c|}{ Máximo } \\
\hline & 2019 & 2020 & 2019 & 2020 & 2019 & 2020 & 2019 & 2020 \\
\hline Santo Tirso & 3.65 & 3.23 & $15.73 \pm 6.03$ & $13.52 \pm 7.37$ & 14.09 & 11.74 & 33.88 & 39.40 \\
\hline Braga-urbano & 24.40 & 9.70 & $56.73 \pm 16.52$ & $27.56 \pm 9.01$ & 55.45 & 26.65 & 101.01 & 54.22 \\
\hline Braga-rural & - & - & - & - & - & - & - & - \\
\hline Guimarães & 14.61 & 4.70 & $44.25 \pm 14.83$ & $28.52 \pm 12.74$ & 43.57 & 25.90 & 77.91 & 63.75 \\
\hline Paços de Ferreira & 3.84 & 8.41 & $14.89 \pm 5.82$ & $18.51 \pm 7.47$ & 13.91 & 19.02 & 30.05 & 33.68 \\
\hline Paredes & 10.58 & - & $30.73 \pm 11.27$ & - & 30.22 & - & 57.06 & - \\
\hline
\end{tabular}


A Figura 2 apresenta diagramas de extremos e quartis calculados para as concentrações de $\mathrm{NO}_{2}$ medidas nas diferentes estações no primeiro semestre de 2019 e 2020, e a tabela 3 apresenta alguns estatísticos sumários. As figuras 3 e 4 mostram a distribuição das concentrações de $\mathrm{NO}$ e NOx, respetivamente, no mesmo período.

Note-se que a estação Braga-rural não efetua medições de $\mathrm{NO}_{2}$ e que a estação de Paredes não apresenta registos para o primeiro semestre de 2020 (Tabela 2).

As cidades de Braga e Guimarães apresentam sempre as concentrações mais elevadas (Tabela 3), provavelmente por serem as cidades de maior população, e portanto, cidades com maior intensidade de tráfego rodoviário. De facto, estudos disponíveis na literatura vêm indicando uma correlação entre a dimensão populacional das cidades e as concentraçôes de poluentes atmosféricos, com as cidades maiores a apresentarem níveis mais elevados de $\mathrm{NO}_{2}$ e $\mathrm{PM}_{10}$ (Orioli et al. 2018), por exemplo.

Em média, as concentrações de $\mathrm{NO}_{2}$ são significativamente $(p<0.0001)$ mais baixas em 2020. Paços de Ferreira é a exceção, registando uma subida significativa ( $p<$ $0.005)$ dos níveis de $\mathrm{NO}_{2}$ em 2020. No entanto, é a cidade que tende a apresentar os valores mais baixos desta aglomeração. 

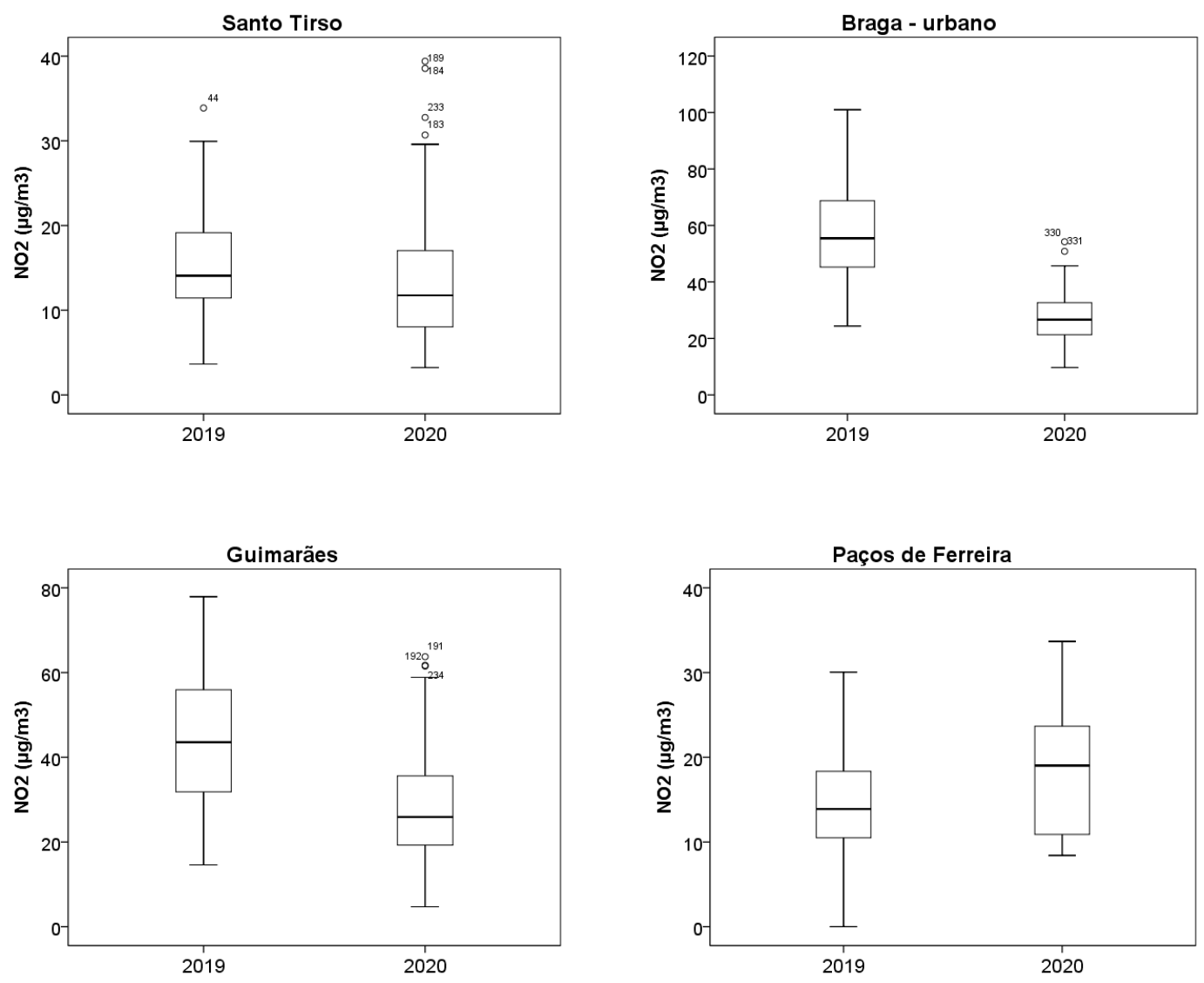

Figura 2. Diagramas de extremos e quartis das médias diárias de $\mathrm{NO}_{2}$ registadas nas estaçốes de Santo Tirso, Braga-urbano, Guimarães e Paços de Ferreira nos primeiros seis meses de 2019 e 2020.

$\mathrm{NO}$ e $\mathrm{NO}_{\mathrm{x}}$ apresentam distribuições semelhantes ao $\mathrm{NO}_{2}$, novamente com uma descida dos valores em 2020 e com a exceção de Paços de Ferreira. No entanto, as diferenças não são estatisticamente significativas $(p>0.05)$ para o NO medido nas estações de Santo Tirso e Paços de Ferreira.

Tal como para o $\mathrm{NO}_{2}$, as cidades de Braga e Guimarães apresentam as concentraçóes mais elevadas de $\mathrm{NO}$ e $\mathrm{NO}_{\mathrm{x}}$ nos dois anos em estudo, um resultado expectável uma vez que o $\mathrm{NO}_{2}$ se forma por oxidação do $\mathrm{NO}$. 

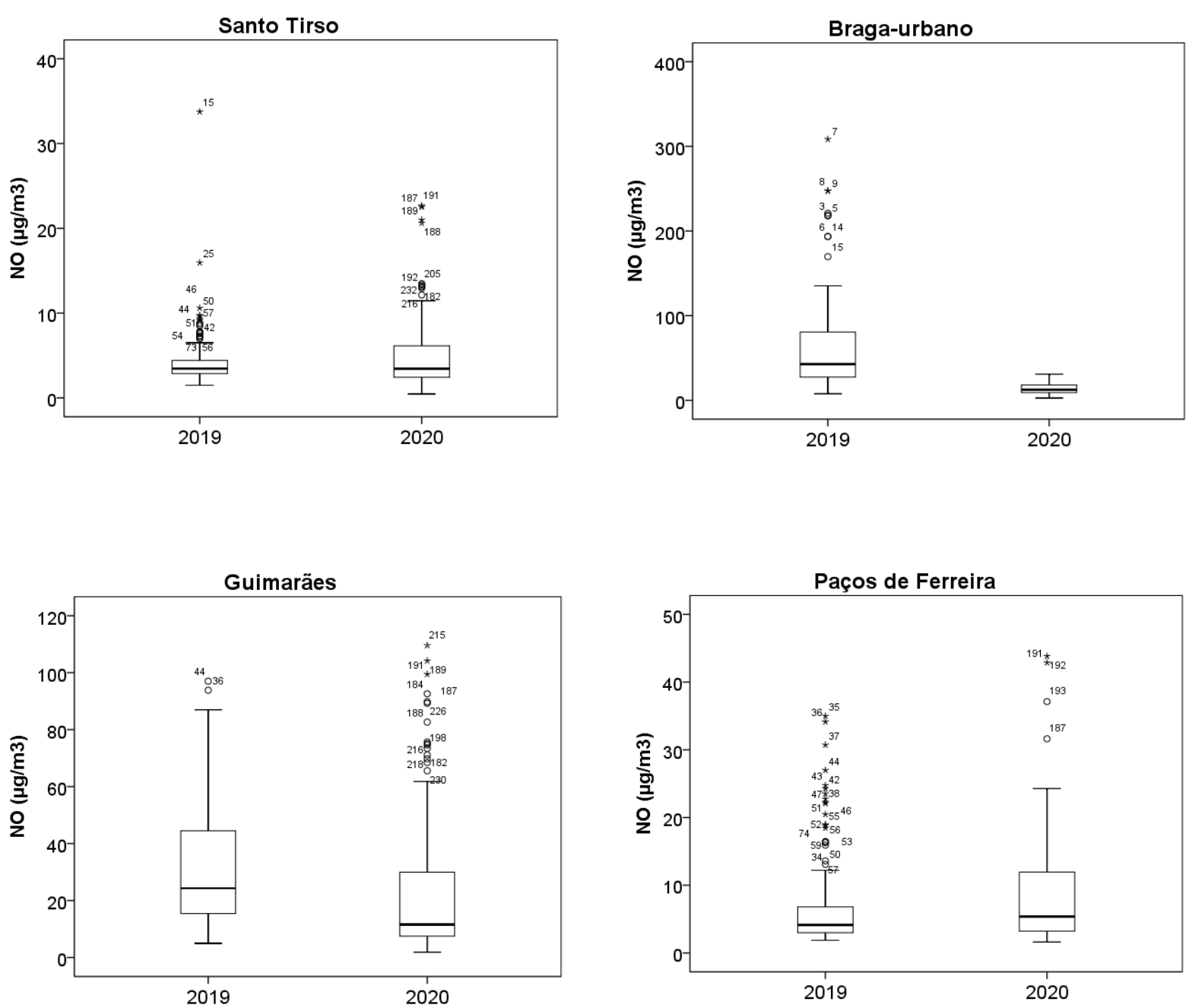

Figura 3. Diagramas de extremos e quartis das médias diárias de NO registadas nas estaçóes de Santo Tirso, Braga-urbano, Guimarães e Paços de Ferreira nos primeiros seis meses de 2019 e 2020.

Para além da significativa diminuição dos valores de $\mathrm{NO}$ e $\mathrm{NO}_{x}$, em Braga-urbano é assinalável a pequena dispersão dos valores em torno da média (Fig. 3 e Fig. 4) que resultou num estreito intervalo de variação das concentrações destes compostos no primeiro semestre de 2020. Considerando que esta estação de medição se situa na Avenida Frei Bartolomeu dos Mártires, um dos principais eixos rodoviários da cidade que, em condiçôes normais, é caracterizado por uma forte heterogeneidade temporal no volume de tráfego, esta pequena variabilidade nos valores de NO pode ser explicada pela drástica diminuição do tráfego automóvel durante a fase de confinamento imposta pelas autoridades. 

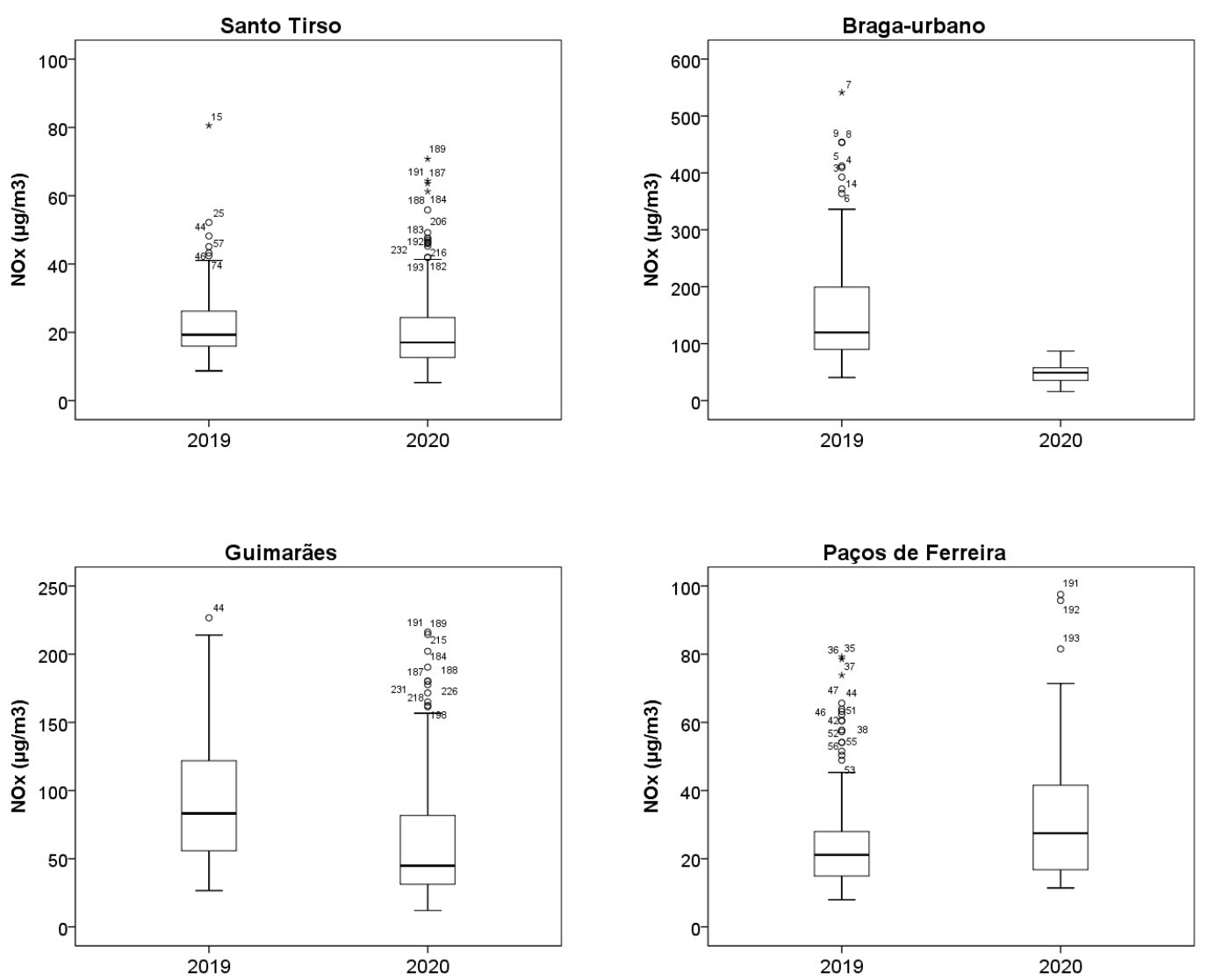

Figura 4. Diagramas de extremos e quartis das médias diárias de $\mathrm{NO}_{\mathrm{x}}$ registadas nas estações de Santo Tirso, Braga-urbano, Guimarães e Paços de Ferreira nos primeiros seis meses de 2019 e 2020.

$\mathbf{O}_{3}$

Pelas suas características químicas, a exposição humana ao $\mathrm{O}_{3}$ ocorre quase que exclusivamente por inalação. Resultados de estudos toxicológicos em animais e estudos epidemiológicos permitem afirmar que existe uma relação causal entre a exposição a curto prazo ao $\mathrm{O}_{3}$ e efeitos respiratórios (Nuvolone et al., 2018).

$\mathrm{Na}$ aglomeração Entre Douro e Minho, apenas as estações de Santo Tirso, Braga-rural e Paços de Ferreira efetuam mediçóes deste poluente (Tabela 2).

A tabela 4 apresenta alguns estatísticos sumários e a figura 5 apresenta a distribuição dos dados das diferentes estações nos primeiros seis meses de 2019 e 2020. 
Os resultados são variáveis entre estações, com Santo Tirso a registar valores significativamente ( $p<0.0001)$ mais elevados em 2020, e as estaçôes de Braga-rural e Paços de Ferreira a registarem valores mais baixos no mesmo ano (Fig. 5). No entanto, nesta última estação a diferença nas concentrações não é significativa ( $p>0.05$ ). Note-se ainda o elevado número de valores anómalos observados na estação de Braga-rural em 2020.

Paços de Ferreira apresenta consistentemente os valores mais altos de $\mathrm{O}_{3}$ (Tabela 4), apesar do decréscimo nas concentrações observado nos primeiros seis meses de 2020.
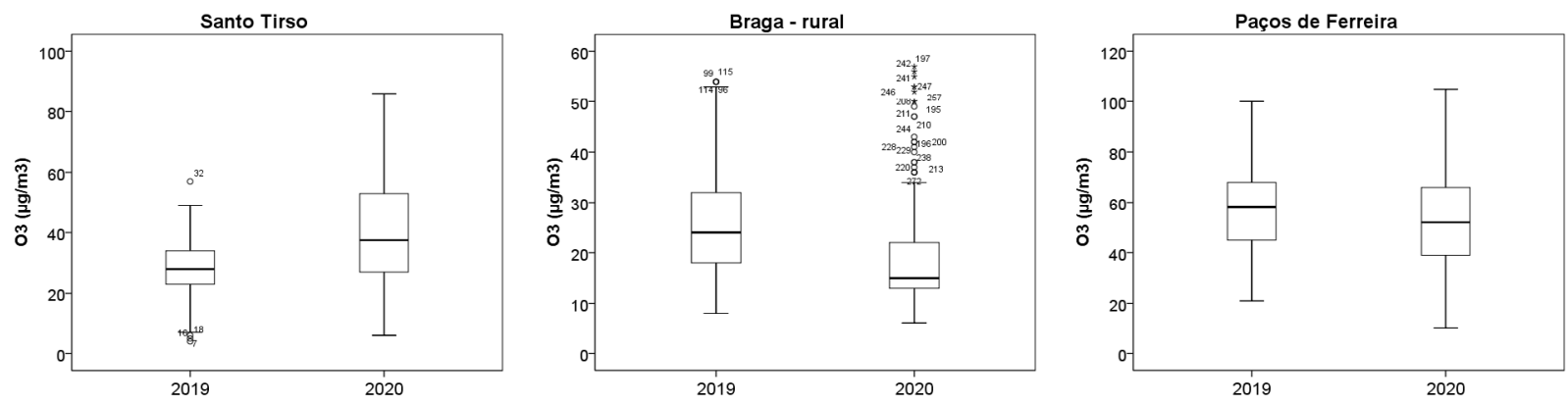

Figura 5. Diagramas de extremos e quartis das médias diárias de $\mathrm{O}_{3}$ registadas nas estaçóes de Santo Tirso, Braga-rural, e Paços de Ferreira nos primeiros seis meses de 2019 e 2020.

Comparando a distribuição dos dados de $\mathrm{NO}_{2}$ (Fig. 2) e $\mathrm{O}_{3}$ (Fig. 5) nas estações de Santo Tirso e Paços de Ferreira, as únicas que registam concentrações dos dois poluentes, verifica-se que estes apresentam uma distribuição inversa, isto é, no ano em que os níveis de $\mathrm{NO}_{2}$ diminuem (Fig. 2) os níveis de $\mathrm{O}_{3}$ aumentam (Fig. 3), e vice-versa. $\mathrm{O}$ aumento nas concentraçóes de $\mathrm{O}_{3}$ observado em Santo Tirso no ano de 2020 pode ser explicado pela diminuição das concentraçóes de $\mathrm{NO}_{\mathrm{x}}$ (Fig. 4). Havendo menores quantidades de $\mathrm{NO}$ a serem emitidas para a atmosfera, menores quantidades de $\mathrm{O}_{3}$ participarão nas reaçôes de formação do $\mathrm{NO}_{2}$ e, consequentemente, as concentraçôes aumentam. Resultados semelhantes foram relatados para as cidades de Nice em França, Roma e Turim em Itália, Valência em Espanha, e Wuhan na China (Sicard et al., 2020). 
Tabela 4. Estatísticos sumários calculados para os dados de $\mathrm{O}_{3}$ referentes aos primeiros seis meses de 2019 e 2020.

\begin{tabular}{|c|c|c|c|c|c|c|c|c|}
\hline \multirow[t]{2}{*}{$\mathrm{O}_{3} \mu \mathrm{g} / \mathrm{m}^{3}$} & \multicolumn{2}{|c|}{ Mínimo } & \multicolumn{2}{|c|}{ Média \pm Desvio Padrão } & \multicolumn{2}{|c|}{ Mediana } & \multicolumn{2}{|c|}{ Máximo } \\
\hline & 2019 & 2020 & 2019 & 2020 & 2019 & 2020 & 2019 & 2020 \\
\hline Santo Tirso & 4.00 & 6.00 & $28.01 \pm 9.62$ & $39.16 \pm 16.30$ & 28.00 & 37.50 & 57.00 & 86.00 \\
\hline Braga-urbano & - & - & - & - & - & - & - & - \\
\hline Braga-rural & 8.00 & 6.00 & $25.58 \pm 11.57$ & $19.73 \pm 11.27$ & 24.00 & 15.00 & 54.00 & 57.00 \\
\hline Guimarães & - & - & - & - & - & - & - & - \\
\hline Paços de Ferreira & 21.00 & 10.00 & $57.91 \pm 16.55$ & $53.90 \pm 19.98$ & 58.00 & 52.00 & 100.00 & 105.00 \\
\hline Paredes & - & - & - & - & - & - & & - \\
\hline
\end{tabular}

Entre os poluentes atmosféricos, o material particulado em suspensão (PM) é amplamente reconhecido como o poluente atmosférico mais importante em termos de efeitos na saúde humana, considerando que muitos estudos epidemiológicos comprovam associações significativas entre a concentração de PM no ar e o impacto adverso na saúde (Boldo et al., 2011).

Embora as seis estaçôes efetuem a monitorização das $\mathrm{PM}_{10}$ atmosféricas, as duas estaçôes localizadas em Braga apresentaram, para os períodos em análise, problemas de funcionamento dos sensores (Tabela 2).

A figura 6 apresenta a distribuição dos dados das diferentes estações para o primeiro semestre de 2019 e 2020, e a tabela 5 apresenta alguns estatísticos sumários. 

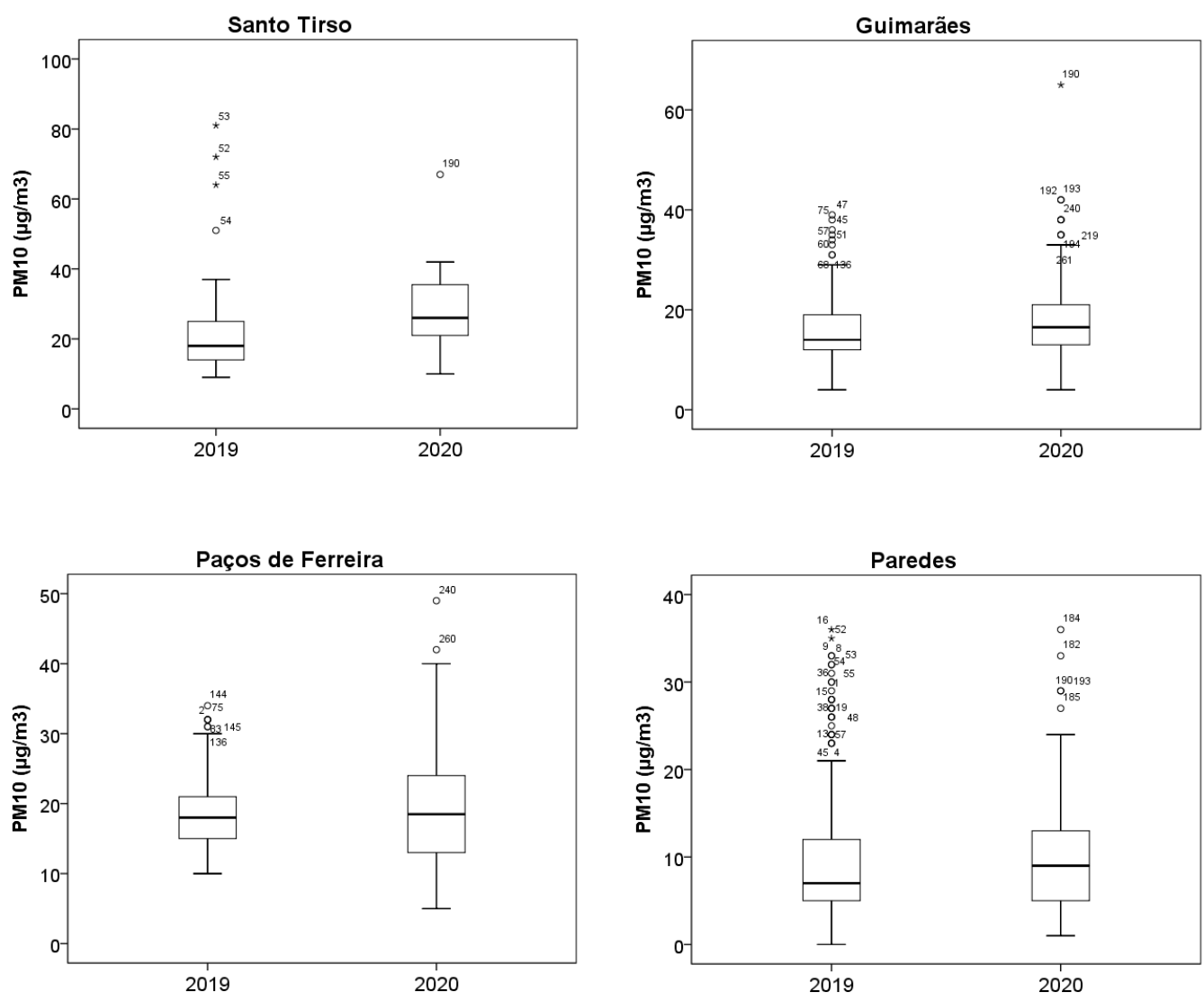

Figura 6. Diagramas de extremos e quartis das médias diárias de $\mathrm{PM}_{10}$ registadas nas estaçóes de Santo Tirso, Guimarães, Paços de Ferreira e Paredes nos primeiros seis meses de 2019 e 2020.

Da tabela 5 observa-se que Paredes regista as concentrações mais baixas em qualquer dos anos enquanto que Santo Tirso apresenta consistentemente as concentrações mais elevadas de $\mathrm{PM}_{10}$.

Nesta região, ao contrário do $\mathrm{NO}_{2}$, a concentração de $\mathrm{PM}_{10}$ no ar ambiente não parece estar diretamente relacionada com a dimensão populacional, uma vez que cidades menores como Santo Tirso e Paços de Ferreira (Tabela 1) apresentam níveis mais elevados de $\mathrm{PM}_{10}$ do que Guimarães, uma cidade com um número de habitantes muito superior. Contudo, este resultado não pode ser confirmado pela ausência de dados para a cidade de Braga (Tabela 2).

Em média, as concentrações de $\mathrm{PM}_{10}$ foram mais elevadas no primeiro semestre do 2020 em todas as estações de medição, mostrando uma distribuição oposta à do 
$\mathrm{NO}_{2}$. No entanto, as diferenças encontradas são significativas $(p<0.0005)$ apenas para as cidades de Santo Tirso e Guimarães.

Tabela 5. Estatísticos sumários calculados para os dados de $\mathrm{PM}_{10}$ referentes aos primeiros seis meses de 2019 e 2020.

\begin{tabular}{|c|c|c|c|c|c|c|c|c|}
\hline \multirow[t]{2}{*}{$\mathbf{P M}_{10} \mu \mathrm{g} / \mathrm{m}^{3}$} & \multicolumn{2}{|c|}{ Mínimo } & \multicolumn{2}{|c|}{ Média \pm Desvio Padrão } & \multicolumn{2}{|c|}{ Mediana } & \multicolumn{2}{|c|}{ Máximo } \\
\hline & 2019 & 2020 & 2019 & 2020 & 2019 & 2020 & 2019 & 2020 \\
\hline Santo Tirso & 9.00 & 10.00 & $22.18 \pm 13.58$ & $27.65 \pm 11.82$ & 18.00 & 26.00 & 81.00 & 67.00 \\
\hline Braga-urbano & - & - & - & - & - & - & - & - \\
\hline Braga-rural & 2.00 & - & $11.43 \pm 10.15$ & - & 8.00 & - & 52.00 & - \\
\hline Guimarães & 4.00 & 4.00 & $16.40 \pm 6.96$ & $18.49 \pm 8.05$ & 14.00 & 16.50 & 39.00 & 65.00 \\
\hline Paços de Ferreira & 10.00 & 5.00 & $18.62 \pm 5.47$ & $19.77 \pm 8.38$ & 18.00 & 18.50 & 34.00 & 49.00 \\
\hline Paredes & 2.00 & 1.00 & $10.67 \pm 8.25$ & $10.02 \pm 6.67$ & 7.00 & 9.00 & 36.00 & 36.00 \\
\hline
\end{tabular}

Ressalve-se que as baixas eficiências obtidas para a maioria das estações (Tabela 2) introduzem um significativo grau de incerteza na análise efetuada à distribuição das $\mathrm{PM}_{10}$ nos dois períodos em estudo.

\section{Distribuição temporal das concentrações dos poluentes}

Os padróes de variação temporal dos poluentes foram avaliados para os primeiros seis meses de 2020. Para uma melhor visualização, cada ponto dos gráficos apresentados nas figuras 7 a 9 representa médias semanais das concentrações do poluente.

$\mathrm{NO}_{2}$

Nas estações de Santo Tirso e Guimarães, após o início do confinamento observa-se uma diminuição gradual das concentraçôes que, no final do mês de junho não 
chegam a atingir os valores registados na fase anterior ao confinamento (AC). Na cidade de Guimarães, o índice de qualidade do ar que em AC oscilava entre o Bom e o Muito Bom, mantém-se estavelmente com a classificação de Muito Bom nas fases CT, CP e PC.

A estação Braga-urbano não registou valores de $\mathrm{NO}_{2}$ na fase anterior ao confinamento, enquanto que a estação de Paços de Ferreira apenas efetuou mediçóes para esta fase (Fig. 7), pelo que não é possível avaliar a evolução temporal do poluente nestas duas áreas. No entanto, com base nos resultados anteriores (Fig. 2) pode-se sugerir uma provável descida dos valores em Braga-urbano e uma subida em Paços de Ferreira durante o confinamento.
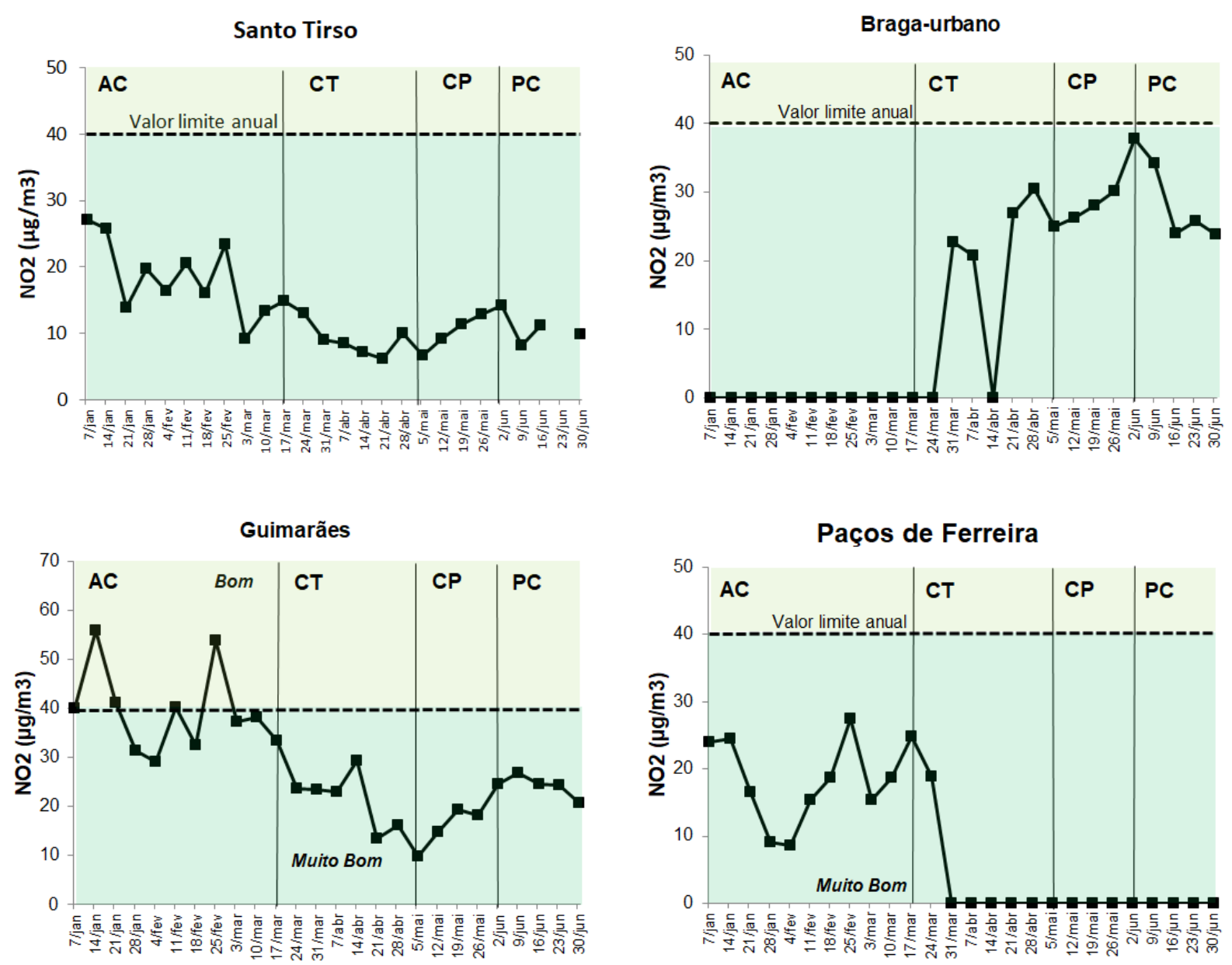

Figura 7. Tendência das concentrações médias diárias de $\mathrm{NO}_{2}$ nas estações de Santo Tirso, Braga-urbano, Guimarães e Paços de Ferreira nas fases anterior ao confinamento (AC), de confinamento total (CT), de confinamento parcial (CP) e após o fim da última fase de desconfinamento (PC). A linha a tracejado indica o valor limite estabelecido na legislação. As diferentes cores refletem os intervalos utilizados na base de dados QualAr para o índice diário de qualidade do ar (verde água-Muito Bom; verde-Bom). 
Em resumo, as concentrações mais baixas de $\mathrm{NO}_{2}$ atmosférico registadas durante os primeiros 6 meses de 2020, quando comparadas com igual período de 2019, parecem ser o resultado direto das medidas impostas pelas autoridades durante o Estado de Emergência.

$\mathbf{O}_{3}$

Em média, as concentraçôes de $\mathrm{O}_{3}$ subiram em Santo Tirso e desceram em Braga-rural durante as fases CT, CP e PC (Fig. 8). Em Paços de Ferreira não é visível uma tendência clara de aumento ou diminuição das concentrações. As concentraçóes mais elevadas observadas durante o confinamento em Santo Tirso estarão certamente relacionadas com menores concentraçóes de $\mathrm{NO}$ e $\mathrm{NO}_{x}$ (não apresentados) registadas no mesmo período. Menores emissóes de $\mathrm{NO}$ implica que menos $\mathrm{NO}_{2}$ seja formado por reação com o $\mathrm{O}_{3}$, e a concentração deste último aumenta.
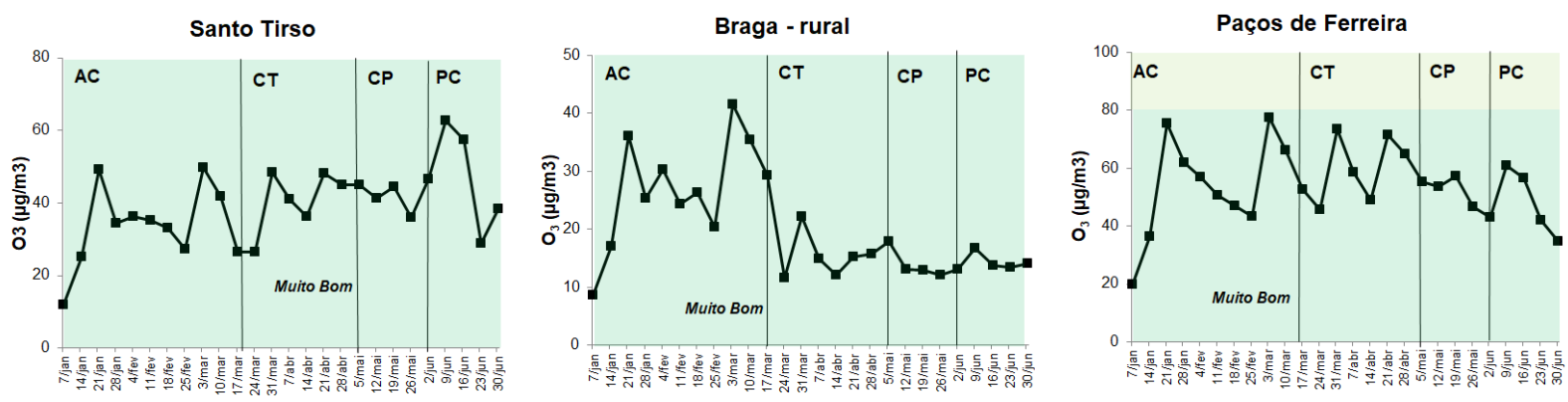

Figura 8. Tendência das concentrações médias diárias de $8 \mathrm{~h}$ nas estações de Santo Tirso, Braga-rural, e Paços de Ferreira nas fases anterior ao confinamento (AC), de confinamento total (CT), de confinamento parcial (CP) e após o fim da última fase de desconfinamento (PC). As diferentes cores refletem os intervalos utilizados na base de dados QualAr para o índice diário de qualidade do ar (verde água-Muito Bom; verde-Bom).

Nestas estaçóes, o índice de qualidade do ar manteve-se consistentemente no intervalo de Muito Bom durante os primeiros 6 meses de 2020, com Paços de Ferreira a registar os valores mais elevados neste período.

Importa salientar que as concentrações médias diárias de $8 \mathrm{~h}$ registadas nestas 3 estaçôes se encontram bem abaixo dos limiares de alerta $\left(240 \mu \mathrm{g} / \mathrm{m}^{3}\right)$ e de informação

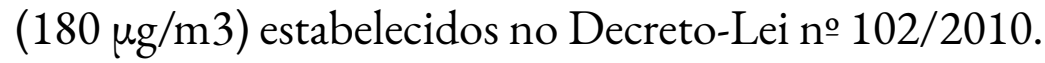


$\mathbf{P M}_{10}$

A baixa eficiência dos dados (Tabela 2) dificulta a identificação do padrão de variação temporal das concentrações de $\mathrm{PM}_{10}$ nas estações de Santo Tirso e Paços de Ferreira (Fig. 9). Nas restantes estações nota-se uma tendência de diminuição das concentrações ao longo das fases CT, CP, e mesmo PC. Em Guimarães, com o confinamento, o índice de qualidade do ar evolui de maioritariamente Bom para Muito Bom. As médias diárias mantêm-se sempre abaixo do valor limite diário estabelecido na legislação, mesmo durante a fase AC.

Comparando com a figura 6 verifica-se que, apesar dos níveis de $\mathrm{PM}_{10}$ terem aumentado entre os primeiros seis meses de 2019 e igual período de 2020, o confinamento parece ter contribuído para uma diminuição das concentraçôes deste poluente, pelo menos nas estações onde foi possível identificar um padrão de variação temporal.
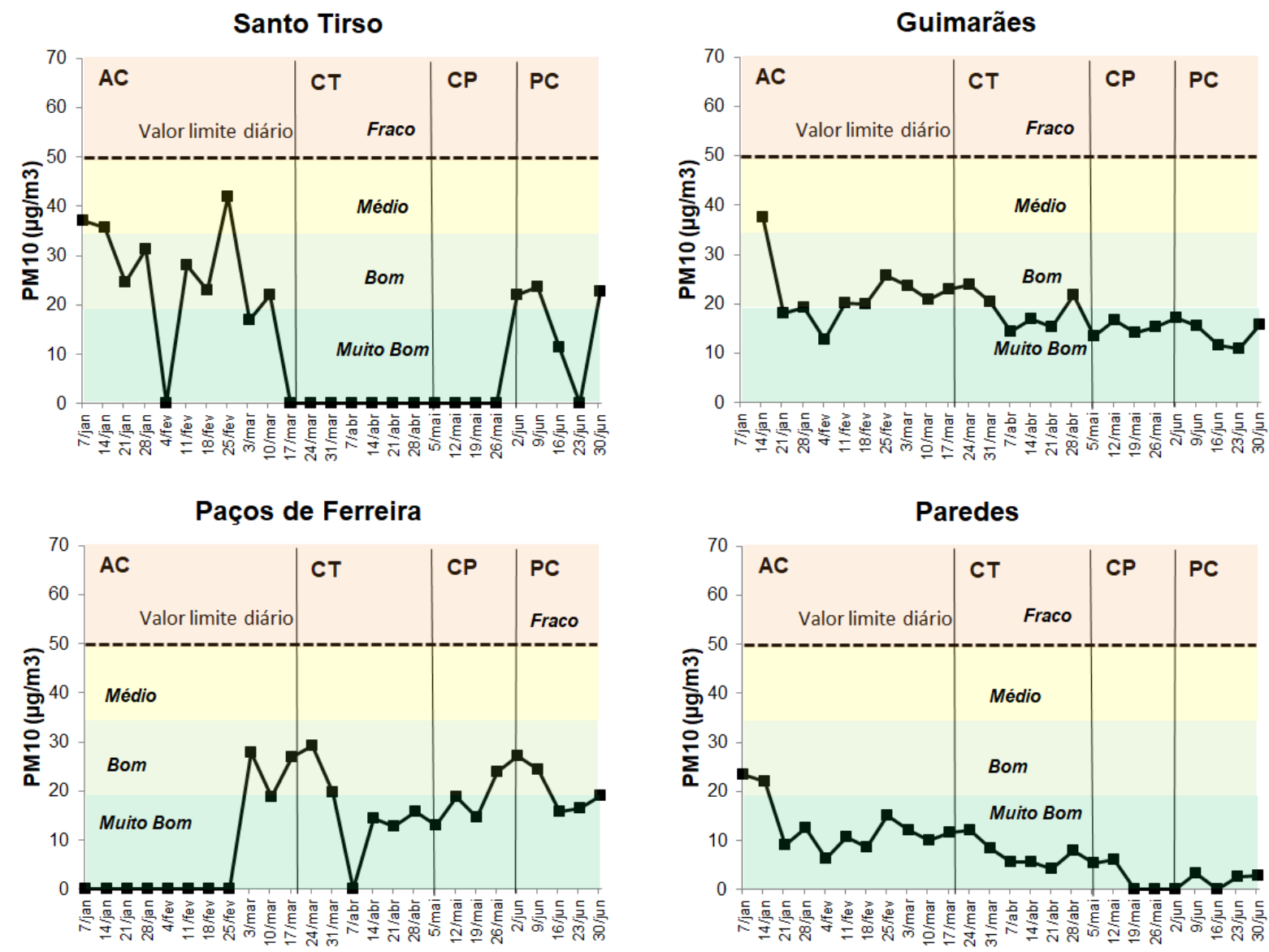

Figura 9. Tendência das concentrações médias diárias nas estações de Santo Tirso, Guimarães, Paredes e Paços de Ferreira nas fases anterior ao confinamento (AC), de confinamento total (CT), de confinamento parcial (CP) e após o fim da última fase de desconfinamento (PC). A linha a tracejado indica o valor limite estabelecido na legislação. As diferentes cores refletem os intervalos utilizados na base de dados QualAr para o índice diário de qualidade do ar (verde água-Muito Bom; verde-Bom; amarelo-Médio; laranja-Fraco). 


\section{CONCLUSÕES}

Neste artigo, pretendeu-se avaliar o efeito do confinamento na qualidade do ar da aglomeração Entre Douro e Minho. No entanto, a baixa eficiência dos dados obtidos pelas seis estaçôes de monitorização, associado ao facto de diferentes estaçôes monitorizarem poluentes diferentes, dificultaram uma análise detalhada do impacto efetivo da imposição do Estado de Emergência na qualidade do ar. Demonstra-se, deste modo, a importância de existirem redes eficientes de medição e registo de parâmetros ambientais, sem os quais não é possível desenvolver estudos científicos que possam auxiliar os responsáveis políticos na tomada de decisão.

A severa limitação do movimento de pessoas após o confinamento e subsequente desconfinamento resultaram numa redução significativa da concentração de $\mathrm{NO}_{2}$ atmosférico, principalmente devido à diminuição do número de veículos em circulação. A redução foi particularmente significativa nas cidades com maior número de habitantes. Apesar da redução do $\mathrm{NO}_{2}$ nas fases $\mathrm{CT}$ e $\mathrm{CP}, \mathrm{o}_{3}$ apresentou um aumento significativo, provavelmente devido à menor concentração de $\mathrm{NO}$ atmosférico. No entanto, são necessários dados adicionais para realizar uma avaliação mais precisa dos efeitos da pandemia na qualidade do ar desta região.

\section{AGRADECIMENTOS}

Este trabalho é financiado por fundos nacionais através da FCT - Fundação para a Ciência e a Tecnologia, I.P., no âmbito dos projetos Refạs UIDB/04683/2020 e UIDP/04683/2020. A autora agradece à Comissão de Coordenação e Desenvolvimento Regional do Norte, e em particular da Dra. Cristina Figueiredo, os dados das médias diárias dos poluentes. 


\section{REFERÊNCIAS BIBLIOGRÁFICAS}

AEA-Agência Europeia do Ambiente (2016). Legislação relativa à qualidade do ar na Europa. Sinais Viver num clima em mudança. Consultado em https://www.eea.europa.eu/pt/sinais-da-aea/sinais-2013/ artigos/legislacao-relativa-a-qualidade-do, acedido em 12/07/2020.

APA-Agência Portuguesa do Ambiente (2020). Ar. Politicas. Consultado em https://apambiente.pt/, acedido em 12/07/2020.

Awasthi, S., (2016). Evaluation of air pollution impact in human health at J.P cement plant, Rewa, (M.P). International Journal of Innovative Drug Discovery, 6, 1-7.

Boldo, E., Linares, C., Lumbreras, J., ..., López-Abente, G. (2011). Health impact assessment of a reduction in ambient PM2.5 levels in Spain. Environment International, 37, 342-348. https://doi.org/10.1016/j. envint.2010.10.004.

Brauer, M. (2010). How much, how long, what, and where: air pollution exposure assessment for epidemiologic studies of respiratory disease. Proc. Am. Thorac. Soc., 7, 111-115. https://doi.org/10.1513/ pats.200908-093RM.

Cohen, A.J., Brauer, M., Burnett, R., Anderson, H.R., Frostad, J., Estep, K., Balakrishnan, K., Brunekreef, B., Dandona, L., Dandona, R., Feigin, V., Freedman, G., Hubbell, B., Jobling, A., Kan, H., Knibbs, L., Liu, Y., Martin, R., Morawska, L., Pope, C.A., Shin, H., Straif, K., Shaddick, G., Thomas, M., van Dingenen, R., van Donkelaar, A., Vos, T., Murray, C.J.L., Forouzanfar, M.H., (2017). Estimates and 25-year trends of the global burden of disease attributable to ambient air pollution: an analysis of data from the Global Burden of Diseases Study 2015. Lancet, 389, 1907-1918. https://doi.org/10.1016/S0140-6736(17)30505-6.

Collivignarelli, M.C., Abbà, A., Bertanza, G., Pedrazzani, R., Ricciardi, P., Miino, M.C. (2020). Lockdown for COVID-2019 in Milan: What are the effects on air quality? Science of the Total Environment, 732, 139280. https://doi.org/10.1016/j.scitotenv.2020.139280.

Dutheil, F., Baker, J. S. \& Navel, V. (2020). COVID-19 as a factor influencing air pollution?. Environmental Pollution, 263, 1-3. https://doi.org/10.1016/j.envpol.2020.114466.

He, L., Zhang, S., Hu, J., Li, Z., Zheng, X., Cao, Y., Xu, G., Yan, M., Wu, Y. (2020). On-road emission measurements of reactive nitrogen compounds from heavy duty diesel trucks in China. Environ. Pollut. 262, 114280. https://doi.org/10.1016/j.envpol.2020.114280.

INE-Instituto Nacional de Estatística (2016). Estatísticas territoriais. Consultado em http://www.ine. pt, acedido a 23/07/2020.

IPMA- Instituto Português do Mar e da Atmosfera (2020). Clima de Portugal Continental. Consultado em https://www.ipma.pt/pt/educativa/tempo.clima/index.jsp?page=clima.pt.xml, acedido a 24/07/2020.

Kastury, F., Smith, E., \& Juhasz, A. L. (2017). A critical review of approaches and limitations of inhalation bioavailability and bioaccessibility of metal(loid)s from ambient particulate matter or dust. Science of the Total Environment, 574, 1054-1074. https://doi.org/10.1016/j.scitotenv.2016.09.056. 
Mahato, S., Pal, S., Ghosh, K.G. (2020). Effect of lockdown amid COVID-19 pandemic on air quality of the megacity Delhi, India. Science of the Total Environment, 730, 139086. https://doi.org/10.1016/j. scitotenv.2020.139086.

Marinho Reis, A. P., Cave, M., Sousa, A. J., Wragg, J., Rangel, M. J., Oliveira, A. R., ... Noack, Y. (2018). Lead and zinc concentrations in household dust and toenails of the residents (Estarreja, Portugal): a sourcepathway-fate model. Environmental Science: Processes and Impacts, 20(9), 1210-1224. https://doi.org/10.1039/ c8em00211h.

Nakada, L.Y.K. \& Urban, R.C. (2020). COVID-19 pandemic: Impacts on the air quality during the partial lockdown in São Paulo state, Brazil. Science of the Total Environment, 730, 139087. https://doi.org/10.1016/j. scitotenv.2020.139087.

Nuvolone, D., Petri, D., Voller, F. (2018). The effects of ozone on human health. Environ Sci Pollut Res, 25, 8074-8088. https://doi.org/10.1007/s11356-017-9239-3.

Orioli, R., Cremona, G., Ciancarella, L., Solimini, A.G. (2018). Association between PM10, PM2.5, NO2, O3 and self-reported diabetes in Italy: A crosssectional, ecological study. PLoS ONE 13(1): e0191112. https:// doi.org/10.1371/journal.pone.0191112.

Otmani, A., Benchrif, A., Tahri, M., Bounakhla, M., Chakir, E.M., El Bouch, M., Krombi, M. (2020). Impact of COVID-19 lockdown on PM10, SO2 and NO2 concentrations in Salé City (Morocco). Science of the Total Environment, 735, 139541. https://doi.org/10.1016/j.scitotenv.2020.139541.

Sicard, P., De Marco, A., Agathokleous, E., Feng, Z., Xu, X., Paoletti, E., Rodriguez, J.J.D., Calatayud, V. (2020). Amplified ozone pollution in cities during the COVID-19 lockdown. Science of the Total Environment, 735, 139542. https://doi.org/10.1016/j.scitotenv.2020.139542.

Song, C., Wu, L., Xie, Y., He, J., Chen, X., Wang, T., ... Mao, H. (2017). Air pollution in China: Status and spatiotemporal variations. Environmental Pollution, 227, 334-347. https://doi.org/10.1016/j. envpol.2017.04.075.

WHO- World Health Organization (2020). WHO director-General's opening remarks at the media briefing on COVID-19. Consultado em https://www.who.int/dg/speeches/detail/who-director-general-s-openingremarks-at-the-media-briefing-on-COVID-19-11-march-2020, acedido em 12/07/2020. 